\title{
A MULTIOBJECTIVE DYNAMIC PARTICLE SWARM OPTIMIZER FOR ENVIRONMENTAL/ECONOMIC DISPATCH PROBLEM
}

\author{
Mostafa A. El-hosseini ${ }^{1}$, Ragab A. El-Sehiemy ${ }^{2}$ and Amira Y. H. \\ 1 Computers engineering and Control Systems Department, Faculty of Engineering- \\ Mansoura University, Email: melhosseini@mans.edu.eg; amirayh@gmail.com \\ 2 Electrical Engineering Departments, Faculty of Engineering-Kafrelsheikh University, \\ Corresponding author; Email: elsehiemy@eng.kfs.edu.eg
}

\begin{abstract}
This paper proposes a multi-objective Dynamic Random Neighborhood PSO (DRN-PSO) dynamic search based optimization algorithm for solving dual security constrained economic load dispatch problem in modern power systems. The proposed algorithm uses dynamically adjusted Inertia weight to balance global exploration and local exploitation. Numerical results were conducted on IEEE 30-bus test systems and compared to other optimization techniques that reported in the literature. The obtained results demonstrate the superiority of the proposed DRN-PSO compared to other optimization techniques. Additional economic benefits with secure settings are fulfilled, while preserving all system constraints within their permissible limits. The proposed algorithm improves the economic issue as well as enhancing the power system operation in the technical point of view with acceptable levels of emissions. So, it can be considered as a promising alternative algorithm for solving problems in practical large scale power systems.

Keywords: constrained economic load dispatch, dynamic random neighborhood, environmental emission, multiobjective, Particle swarm optimization, transmission security.

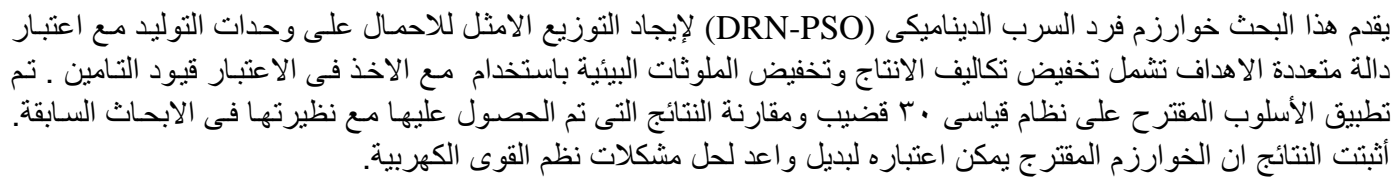

\section{INTRODUCTION}

Developing the search-based optimization algorithms for power system problems has become in the focus of power system developer due to the dramatic variation in fuel costs and the increased concerns of environmental impacts. Power system optimization problems including constrained economic load dispatch (CELD) have complex and nonlinear characteristics with heavy nonlinear equality and inequality constraints. The problem of economic dispatch of electric power generation aims at meeting the load demand at minimum operating cost while satisfying all unit and system equality and inequality constraints. This is done by obtaining the optimum scheduling of the committed generating unit outputs $[1,2]$. The CELD problems have non-convex objective functions with nonlinear equality and inequality constraints. In practice, real input-output characteristics present higher order nonlinearities and discontinuities due to valve-point loading effects caused by the sharp increase in losses when steam admission valves are first opened. Generally, non- convexities arise from valve points or combined cycle units, zones of prohibited operation of unit, and nonlinear power-flow equality constraints [3].

As a result of these characteristics, it is much harder to find the global optimum using any mathematical algorithms. Traditionally, electric power systems aim at operating in such a way that the total fuel cost is minimized regardless of the emission produced in the system. However, an increased public awareness regarding the harmful effects of atmospheric pollutants on the environment has been noticed. The importance of environmental impacts and the passage of the Clean Air Act Amendments of 1990 forced the utilities to adapt their design and operational strategies in order to reduce pollution and atmospheric emissions of the thermal power plants [4-6].

Recently, the dramatic growing of fuel costs and the increased concerns of environmental issues of power generating units present early alarms for the necessity of continuous improvement of optimization methodologies for solving CELD problems efficiently. From power system operation point of 
view, it is necessary to minimize both emission impacts and generation costs simultaneously. The CELD problem may be formulated as multiobjective constrained nonlinear problem. The decision-maker wants to select one optimization scheme to solve the CELD problem. However, the selection of the optimal independent scheme, for solving the CELD problem, must take into account the following considerations [7]. These are the characteristics, types, models of available generation units, both of operation and maintenance costs, the technical and operational constraints, equipment capabilities and transmission line limits, and the reliability of the units for operational points.

Different optimization techniques have been reported in the literature pertaining to the CELD problem. Classical methods, such as the lambda iteration method and gradient method have been applied to solve the Economic Load Dispatch (ELD) problems. However, unfortunately, these methods are not feasible in practical power systems owing to the nonlinear characteristics of the generators and nonsmooth cost functions. Consequently, many powerful mathematical optimization techniques that are fast and reliable, such as non-linear programming and dynamic programming have been employed to solve the ELD problems. However, due to the nondifferential and non-convex characteristics of the cost functions, these methods were also unable to locate the global optima [7]. Meanwhile, convex CELD problems are efficiently solved through traditional local search algorithms such as lambda iteration (which ignores network constraints) [1] and linear programming [2].

Modern heuristics optimization techniques were considered as practical tools for non-linear optimization problems [8]. Particle swarm optimization (PSO) $[9,10]$ marks one of the most popular classes of nature-inspired optimizers and has its roots in artificial life and social psychology. PSO is a population based stochastic optimization technique developed by Eberhart and Kennedy in 1995 and inspired by social behavior of bird flocking or fish schooling [9]. The PSO method was originally intended for simulating the social behavior of a bird flock, however the algorithm was simplified and it was realized that the individuals (typically called particles) were actually performing optimization. PSO has an uncountable number of variants of the basic algorithm. These include theoretical and empirical investigations of the dynamics of the particles, parameter selection and control, and applications of the algorithm to a wide spectrum of real world problems from diverse fields of science and engineering [11-17].

Recently, PSO has been successively applied to various fields of power system optimization problems such as for economic dispatch problem considering generation constraint [8], for minimizing the non-smooth cost function of economic dispatch problem [18, 19], scheduling the generation outputs considering lagrangian relaxation method [20], reactive power and voltage control [21-22], optimal design of power system stabilizer [23], optimal power flow [24], state estimation [25], unit commitment problem [26] and for reactive power control [27].

The multi-objective generation dispatch using PSO with multiple fuel option were presented in [28] while, in [29], the multi-objective generation dispatch using PSO was presented for electricity markets [30] presented a procedure using PSO for obtaining the optimal design of a neuro-sliding mode controller for the transient stability enhancement of multi machine power systems with unified power flow controller (UPFC).

This paper is concerned with solving the CELD problem considering the emission minimization as well as operating cost as multiobjective problem. The considered problem is solved using DRN-PSO algorithm and the obtained results are compared to those reported in the literature. The tested case study is the standard IEEE 30 bus test system. The rest of the paper is organized as follow: section 2 presents the related work, section 3 demonstrates the problem formulation, section 4 presents the proposed algorithm, and finally section 5 describes the tested case studies with a comparative analysis to previous work reported.

\section{PSO BACK GROUND AND RELATED WORK}

\section{a) Traditional PSO algorithm}

In the PSO method the particles are initially placed at random positions in the search-space, moving in randomly defined directions. The direction of a particle is then gradually changed so it will start to move in the direction of the best previous positions of itself and its peers, searching in their vicinity and hopefully discovering even better positions with regard to some fitness measure. Each particle is treated as a point in an n-dimensional space. The ith particle is represented as $x_{i}=\left(x_{i 1}, x_{i 2}, \ldots, x_{i n}\right)$. The best previous position of the ith particle is recorded and represented as $p_{i}=\left(p_{i 1}, p_{i 2}, \ldots, p_{i n}\right)$. The index of the best particle among all the particles in the population is represented by the subscript $g$. The rate of the position change (velocity) for particle $i$ is represented by $v_{i}=\left(v_{i 1}, v_{i 2}, \ldots, v_{i n}\right)$. The particles are manipulated according to the following equations [10]:

$$
\begin{aligned}
& v_{i d}=w_{i} v_{i d}+c_{1} \operatorname{rand}()\left(p_{i d}-x_{i d}\right)+ \\
& c_{2} \operatorname{rand}()\left(p_{g d}-x_{i d}\right) \\
& x_{i d}=x_{i d}+v_{i d}
\end{aligned}
$$


where $d$ is the dimension $(1 \leq d \leq n), c_{1}$ and $c_{2}$ are positive constants, rand ( ) is a random function in the range $[0,1]$, and $w$ is the inertia weight.

The pseudo code of the procedure is as:

While maximum iterations or minimum error criteria are not attained

For each particle

Initialize particle

END

Do

For each particle

Calculate fitness value

If the fitness value is better than the best fitness value (pbest) in history set current value as the new pbest

End

Choose the particle with the best fitness value of all the particles as the gbest

For each particle

Calculate particle velocity according equation (1)

Update particle position according equation (2)

End

Particles' velocities on each dimension are clamped to a maximum velocity $V \max$. If the sum of accelerations would cause the velocity on that dimension to exceedVmax, which is a parameter specified by the user, then the velocity on that dimension is limited to Vmax [10].

b) Variants of PSO algorithm

Similar to other population-based optimization techniques, convergence speed and global search ability are the two critical criteria for evaluating the performance of PSO algorithms. In the original PSO, all particles learn from $g B e s t$ in updating velocities and positions. Hence the algorithm exhibits a fastconverging behavior. But on multimodal problems, a gBest located at a local optimum may trap the whole swarm and lead to premature convergence. Various variants of PSO algorithm have been proposed to improve the performance of PSO for global optimization. The existing PSO variants can be mainly classified into the following four categories as shown in Fig.1.

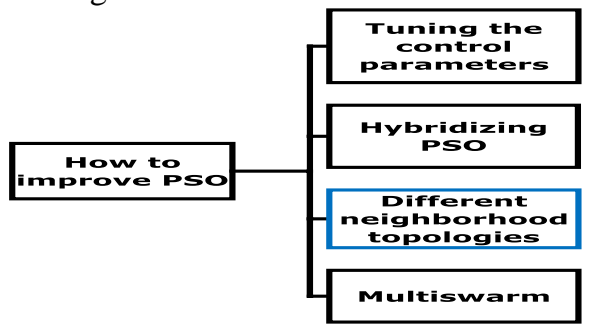

Fig. 1 Different methods to improve PSO performance
As can be seen from Fig.1, different neighborhood topologies aim at increasing diversity by defining neighborhood topologies. The neighborhoods topologies are used to guarantee the convergence [31]. When it is improperly used they will impact the diversity of the particles. The premature problems are solved by making a choice on neighborhoods topologies.

The velocity update rule eq(1) illustrates that the third part only directs the current particle's search directions partially. If the current global best position introduces the directions which do not lead to the convergence point, a wrong direction message will be created. Especially, if the current global best particle catches up with a local optimum and leads global swarm concentrated to that position, the wrong direction message also lead to premature phenomenon.

Kennedy and Mendes investigated the effects of various population topologies on PSO to seek a better structure that performs well on a variety of test problems [15]. They denoted the local version PSO with the ring topology and the von Neumann topology by RPSO and VPSO, respectively. Usually, a large neighborhood is better for simple optimization problems, and a small neighborhood is more suitable for complex multimodal problems [15]. To improve the robustness of PSO, time-varying structures have also been proposed [32], [33].

In order to enhance the impacts of neighborhoods and keep the particle apart from gbest in early iterations, three neighborhood topologies are carried in experiments [34]:

(1) One step forward-backward topology,

(2) Two steps forward-backward topology,

(3) Stochastic one step topology

Kennedy designed four different population topologies, including circle, wheel, star, and random. The reported results showed that population topologies with fewer connections might perform better on highly multimodal problems, while highly interconnected populations would be better for unimodal problems [35]. Suganthan [32] proposed a variable neighborhood operator, where during the initial states of the optimization, the neighborhood is the individual particle itself. As the number of generation increases, the neighborhood is gradually extended to include all particles.

Mendes et al. [36] proposed a fully informed PSO algorithm (FIPS), in which the neighbors of each particle, instead of pbest and gbest, are used to update the velocity. Peram et al. [37] developed the fitness-distance-ratio based PSO (called FDR-PSO), in which each particle is attracted towards the best previous positions visited by its neighbors.

$\mathrm{Hu}$ and Eberhart [33] used dynamic neighborhood PSO to solve multi-objective optimization problems. In each generation, after calculating distance to every 
other particle, each particle finds its new neighbors. Among the new neighbors, each particle finds the local best particle as the (LBEST). Ghosh et al. [38] presented a probabilistic analysis of the particle interaction and information exchange in aLBEST PSO with variable random neighborhood topology.

Suganthan et al. [32] proposed to replace the GBEST component by LBEST solution. They dynamically increase the neighborhood in order to change the $\angle B E S T$ from PBEST to GBEST with increasing number of iterations. The neighborhood may be defined in two different ways. The simplest and fastest method is to consider particles just above and below a specific particle for which a neighborhood is sought. Alternatively, it could calculate distances between particles and choose a fraction of particles that are close to the particle for which a neighborhood is sought. This fraction can be increased gradually to include all particles during final stages of search process. This method can be computational intensive, if the dimension of the parameter space is large.

When exploring large problem spaces, optimization algorithms must effectively balance exploration and exploitation. Generally, it is wise to first make a broad survey of the space, and then focus effort on the regions of the space that look most promising.

\section{PROBLEM FORMULATION}

The non-linear CELD problem of finding the optimal combination of power generation, which minimizes the total fuel cost function of each generator while satisfying the total required demand, can be mathematically stated as a quadratic function. The generators cost curves are represented by quadratic functions with sine components. The superimposed sine components represent the rippling effects produced by the steam admission valve opening. The total $\$ / \mathrm{h}$ fuel cost considers the non-smooth valve point effects can be modeled as [2-4]:

$$
\begin{aligned}
& \min F_{t}=\sum_{i=1}^{N G} f_{i}\left(P G_{i}\right)=\sum_{i=1}^{N G}\left(a_{i}+b_{i} P G_{i}+\right. \\
& \left.c_{i} P G_{i}^{2}\right)+\left|d_{i} \sin \left[e_{i} P G_{i}-P G_{i}^{\text {min }}\right]\right|
\end{aligned}
$$

Where, $F_{t}$ : is the non-linear objective function defining the total power generation cost of the system. $a_{i}, b_{i}$ and $c_{i}$ are the coefficients of power generation cost function and $d_{i}$, and $e_{i}$ are the coefficients of non-smooth operation of valves. $N G$ is the number of generation buses. The main objective is to minimize value of the $F_{t}$.

The second objective aims at minimizing the emission effects. The atmospheric pollutants such as sulpher oxides and nitrogen oxides caused by fossil fueled thermal units can be modeled separately. However, for comparison o $\min E_{t}=\sum_{i=1}^{N G} g_{i}\left(P G_{i}\right)=\sum_{i=1}^{N G} 1^{-2}\left(\alpha_{i}+\right.$

$\left.\beta_{i} P G_{i}+\gamma_{i} P G_{i}^{2}\right)+\left|\zeta_{i} \exp \left[\lambda_{i} P G_{i}\right]\right|$

Where, $\alpha \mathrm{i}, \beta \mathrm{i}, \gamma \mathrm{i}, \xi \mathrm{i}$ and $\lambda \mathrm{i}$ are the coefficients of power generation emissions. The previous objective functions are subjected to the following constraints:

As the generators real and reactive power outputs should be equal to the total load demand and transmission line losses, this constraint can be expressed as:

$\sum_{i=1}^{N B} P_{G i}=\sum_{j=1}^{N L} P_{D j}+P_{\text {Loss }}$

Where, $P_{G i}$ is the power generation at bus $i, P_{D j}$ is the load demand at load bus $j, N L$ is the number of load buses and $P_{\text {Loss }}$ is the total power losses in the system.

The generation hard constraints include generator voltages, real power outputs, these constraints are defined as hard constraints as they are restricted by their physical lower and upper limits. The generation constraints can be simulated as:

$$
\begin{gathered}
P_{G i \min } \leq P_{G i} \leq P_{G i \max } \\
Q_{G i \min } \leq Q_{G i} \leq Q_{G i \max } \\
V_{i \min } \leq V_{i} \leq V_{i \max }
\end{gathered}
$$

where $P_{G i \text { min }}$ : minimum power generated, and $P_{G i \text { max }}$ : maximum power generated.

A mathematical formulation of the security CELD problem would require a very large number of constraints to be considered. However, for typical systems the large proportion of lines has a rather small possibility of becoming overloaded. The CELD problem should consider only the small proportion of lines in violation, or near violation of their respective security limits which are identified as the critical lines. We consider only the critical lines that are binding in the optimal solution. The line flow of the $\mathrm{j}^{\text {th }}$ line is expressed in terms of the control variables $P_{G i}$, by utilizing the generalized generation distribution factors (GGDF) [24] and is given below.

$T_{j}\left(P_{G}\right)=\sum_{i=1}^{n}\left(D_{j i} P_{G i}\right)$

Where, $D_{j i}$ is the generalized GGDF for line $\mathrm{j}$, due to generator $\mathrm{i}$ and $T_{j}\left(P_{G}\right)$ is the real power flow.

Using Eq. (7), the power system operator is allowed to ramp the power generation and transmission lines constraints corresponding to the amount of reserve that is able to prepare sufficient preventive control actions as $[7,23]$. For secure operation, the transmission line loading $S_{l}$ is restricted by its upper limit as:

$S_{l} \leq S_{l \text { max }}, l=1,2, \ldots, n_{L}$

Where $\boldsymbol{n}_{\boldsymbol{L}}$ is the number of transmission line.

3.1. Ramp rate limit Constraints

The power generated by the generator $i$ in certain interval may not exceed that of previous interval by 
more than a certain amount, (the up-ramp limit) anstep 2: Setting Initial Swarm

neither may it be less than that of the previous interval by more than some amount, (the down-ramp limit of the generator). Additional generation hard constraints are restricted by their physical ramp rate limits. The ramp rate constraints can be simulated as:

$D R G_{i}{ }^{\min } \leq P G_{i}-P G_{i}{ }^{(0)} \leq U R G_{i}{ }^{\max }$

Where, $U R G_{i}{ }^{\max }$ and $D R G_{i}{ }^{\text {min }}$ are the maximum and minimum of ramp rate for power generation at bus $i$ respectively and $P G_{i}{ }^{(0)}$ is the initial value. These rates are considered around $10 \%$ around the initial generation outputs $\left(P G_{i}\right)$.

\section{PROPOSED MULTI-OBJECTIVE DYNAMIC RANDOM NEIGHBORHOOD PSO (DRN- PSO)}

\section{a) Proposed algorithm}

The proposed algorithm DRN-PSO has many features incorporated to the simple PSO that make the algorithm not getting stuck in local optimst converges faster, and be able to cover all the search space. Traditionally, PSO takes certain predetermined particles as the neighbors. The number of neighbors or the size of the neighborhood wAtep affect the convergence speed of the algorithm. DRNPSO presents new form of dynamic random neighborhood which enables each particle to change its neighborhood during searching for the optimal solution. This feature helps in increasing the swarm diversity. When using DRN-PSO, it is possible for the magnitude of the velocities to become very large. In addition to enforcing search-space boundaries after updating a particle's position, it is also customary to impose limitations on the distance a particle can move in a single step. This is done by bounding a particle's velocity $\vec{v}$ to the full dynamic range of the search-space, so the particle can at most move from one search-space boundary to the other in one step.

Performance can suffer if $V \max$ is inappropriately set. Particles' velocities are clamped to a maximum velocityVmax, which serves as a constraint to control the global exploration ability of particle swarm this paper control the growth of velocities by a dynamically adjusted inertia factor. Initially the values of the velocity vectors are randomly generated with the range, [Vmin Vmax] where Vmax is the maximum value of velocity that can be assigned to any particle and $V \min =-V \max$. The proposed algorithm is detailed as:

\section{Step 1: Load System Data}

Load IEEE 30 bus system data; Fuel cost parameters $(\boldsymbol{a}, \boldsymbol{b}, \boldsymbol{c}, \boldsymbol{d}, \boldsymbol{e})$ for each generator, generator power limits, generator emission coefficients $(\boldsymbol{\alpha}, \boldsymbol{\beta}, \boldsymbol{\gamma}, \boldsymbol{\zeta}, \boldsymbol{\lambda})$, and power flow coefficient
Number of particles; No. of Particles $=20$

Number of neighbors: NoOfNeighbors $=\mathbf{5}$

Number of Dimension: DimNum $=\mathbf{6}$

Number of cycles: $\boldsymbol{C y c N u m}=\mathbf{2 0}$

Maximum Inertia Weight; $w \max =0.9$

Minimum Inertia Weight wmin $=0.4$

pbest - the best solution achieved so far by that particle.

gbest - The best value obtained so far by any particle in the neighborhood of that particle

Initialize particles with random position (candidate solutions) in the range of generator power ranges, and Initialize particle with zero velocity

if < stopping criteria not met > do

\section{ep 3: Fitness function}

For each individual $x \in N$ : calculate fitness $f(x)$; (Fuel cost and/or emission minimization)

\section{tep 4: Constraint handling}

If any one of the $\boldsymbol{p g}$ is outside the range, i.e. constraint violation then punish it, If the power flow in any transmission line is exceeded the secure limit then punish it, and Check power balance if violated then punish it

5: Update pbest

For each particle;

Set pbest as the best position of particlex; If $\boldsymbol{f}(\boldsymbol{x})<f(\boldsymbol{p b e s t})$ then $\boldsymbol{p b e s t}=\boldsymbol{x}$

Update $g$ best; best neighbor for each particle

Every particle is assigned randomly a neighborhood that is consisted of NoOfNeighbors $=\mathbf{5}$ particles, after evaluating each particle fitness in the neighborhood, the best fitness in the neighborhood of each particle is assigned to $\boldsymbol{g}$ best

7: Update velocity and position

For each particle;

Choose the best value of Inertia Weight; $\boldsymbol{w}$ $W=\boldsymbol{W m a x}-((\boldsymbol{W} \max -\boldsymbol{W} \min ) / C y c N u m) \times i$ For every dimension

$\operatorname{Vmax}(:, j)=0.005 *(X \max (:, j)-X \min (:, j))$;

$\operatorname{Vmin}(:, j)=-\operatorname{Vmax}(:, j)$

$$
\begin{aligned}
& \boldsymbol{v}(:, j) \\
& =\min (\max ((\boldsymbol{W} * \boldsymbol{v}(:, j) \\
& +\underbrace{1.4 * \operatorname{rand} *(p i(:, j)-x(:, j))}_{\text {Personal Influence }}+
\end{aligned}
$$

$\underbrace{1.4 * \operatorname{rand} *(\boldsymbol{p g}(j)-x(:, j))}_{\text {Social Influence }}), \operatorname{Vmin}(:, j)), \operatorname{Vmax}(:, j)$

End

End

$x=\min (\max ((x+v), X \min ), X \max ) ;$

Go to Step 3

Otherwise

Print out the generator power along with the considered objective function,

Exit

\section{b) Handling of constraints}

The proposed DRN-PSO deals efficiently with the inequality constraints. The equality constraints are treated as close to inequality constraints as given below: 


$$
\sum P_{g}-\sum \text { Load }- \text { Losses }>\epsilon
$$

Where, $\epsilon$ refers to the convergence degree

\section{c) Handling of conflicting objectives}

The typical CELD problem can be formulated as a bi-criteria optimization model. The two conflicting objectives, i.e., fuel cost and pollutants emission, should be minimized simultaneously while fulfilling certain system constraints. The CELD problem has two objective functions fuel cost minimization and emission minimization. These two objectives are conflicted in nature. The mathematical formulation of multiobjective CELD problem minimizes objective functions Equations (3) and (4) while satisfying system operating constraints.

The weighted-sum method transforms a set of objectives into a single objective by pre-multiplying each objective with user-supplied weight. The weight of an objective is usually chosen in proportion to the objective's relative importance to the problem. It is likely that each objective function takes different magnitude as in the combined economicenvironmental power dispatch. Therefore, setting up an appropriate weight factor depends on the scaling of each objective function. It is the usual practice to choose weights such that their sum is equal to one.

The combined CELD problem can be formulated as follows:

$$
F=w_{1} \times F_{1}(F)+w_{2} \times F_{2}(E t)
$$

Where, $F$ refers to the combined objective function involves fuel cost and emission; $\mathrm{w}_{1}$ and $\mathrm{w}_{2}$ is the weighing factors of the two objective functions.

\section{CASE STUDIES}

\subsection{Test Systems}

In order to validate and to show the effectiveness of the proposed approach for solving the CELD problems using a DRN-PSO, the proposed approach is tested with the standard IEEE 30 bus test system whose single line diagram is shown in Fig. 2 [39]. The test system constitutes 41 lines and six generators located at buses 25-30. Tables 1 and 2 show the cost and emission coefficients of the sixgenerators studied system with their minimum and maximum limits of power, respectively. The upper and down ramp rate are considered with $\pm 10 \%$.

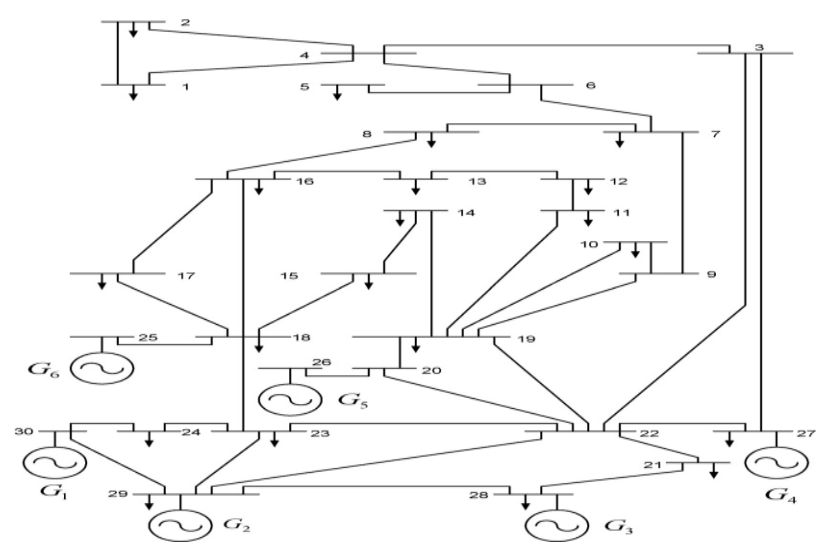

Fig. 2 Single-line diagram of IEEE 30-bus test system [39]

Table1 Generation limits and cost coefficients

\begin{tabular}{|c|c|c|c|c|c|c|c|}
\hline Generator & $\begin{array}{c}\text { Min } \\
\text { MW }\end{array}$ & $\begin{array}{c}\text { Max } \\
\text { MW }\end{array}$ & $\begin{array}{c}\mathrm{a} \\
\$ / \mathrm{MW}^{2}\end{array}$ & $\begin{array}{c}\mathrm{b} \\
(\$ / \mathrm{MW})\end{array}$ & $\begin{array}{c}\mathrm{c} \\
\$\end{array}$ & $\begin{array}{c}\mathrm{d} \\
\$\end{array}$ & $\begin{array}{c}\mathrm{e} \\
\mathrm{MW}^{-1}\end{array}$ \\
\hline $\mathrm{G}_{1}$ & 0.05 & 0.5 & 10 & 200 & 100 & 32.4 & 0.047 \\
\hline $\mathrm{G}_{2}$ & 0.05 & 0.6 & 10 & 150 & 120 & 32.4 & 0.047 \\
\hline $\mathrm{G}_{3}$ & 0.05 & 1 & 20 & 180 & 40 & 32.4 & 0.047 \\
\hline $\mathrm{G}_{4}$ & 0.05 & 1.2 & 10 & 100 & 60 & 23.4 & 0.063 \\
\hline $\mathrm{G}_{5}$ & 0.05 & 1 & 20 & 180 & 40 & 24 & 0.063 \\
\hline $\mathrm{G}_{6}$ & 0.05 & 0.6 & 10 & 150 & 100 & 24 & 0.063 \\
\hline
\end{tabular}

Table 2 Generator emission coefficients

\begin{tabular}{|c|c|c|c|c|c|c|}
\hline Coefficient & $\mathrm{G}_{1}$ & $\mathrm{G}_{2}$ & $\mathrm{G}_{3}$ & $\mathrm{G}_{4}$ & $\mathrm{G}_{5}$ & $\mathrm{G}_{6}$ \\
\hline$\alpha$ & 4.091 & 2.543 & 4.258 & 5.326 & 4.258 & 6.131 \\
\hline$\beta$ & -5.554 & -6.047 & -5.094 & -3.550 & -5.094 & -5.555 \\
\hline$\gamma$ & 6.490 & 5.638 & 4.586 & 3.380 & 4.586 & 5.151 \\
\hline$\xi$ & $2.0 \mathrm{E}-4$ & $5.0 \mathrm{E}-4$ & $1.0 \mathrm{E}-6$ & $2.0 \mathrm{E}-3$ & $1.0 \mathrm{E}-6$ & $1.0 \mathrm{E}-5$ \\
\hline$\lambda$ & 2.857 & 3.333 & 8.000 & 2.000 & 8.000 & 6.667 \\
\hline
\end{tabular}

\subsection{Studied cases}

To assess the efficiency of the proposed DRN-PSO, it has been applied to CELD problems where the objective functions can be either smooth or nonsmooth. The studied cases can be classified under the following three categories:

Case 1: Minimization of the fuel costs only.

Case 2: Minimization of the emissions only.

Two additional joint fuel cost and emission minimization simultaneously are considered as:

Case 3: both objectives are optimized simultaneously with equal priority.

Case 4: both objectives are optimized simultaneously using weighted sum approach.

The proposed optimization approaches compared with the results obtained with multi-objective evolutionary algorithms like non-dominated sorting genetic algorithm (NSGA) [40], niched Pareto genetic algorithm (NPGA) [40], strength Pareto evolutionary algorithm (SPEA) [40], and multiobjective fuzzy based on particle swarm optimization algorithm [18], Modified Shuffled Frog Leaping Algorithm (MSFLA) [41] and an improved real coded genetic algorithm (RCGA) [42]. 


\subsection{Results \& Discussion}

\subsubsection{Case 1: Fuel cost minimization}

Table 3 shows the CELD solution solved through the proposed DRN-PSO algorithm compared to real coded genetic algorithm (RCGA) [42] for Case 1. The proposed method gets different load dispatch settings for the studied cases. From table 3, the fuel cost is $591.1517 \$ / \mathrm{hr}$ while the pollutant emission is 0.215 ton/hr. Compared to RCGA, the fuel cost is improved with total reduction of $24.3965 \$ / \mathrm{hr}$. Also, Table 3 presents the evaluation of the proposed algorithm in terms of mean, best and worst values for 100 runs and the related standard deviation for each case using both optimization methods. Fig. 3 illustrates the best fuel cost against run number. The proposed DRN-PSO algorithm improves the convergence characteristics for case las can be noticed from swarm 10 and 15, Fig. 4.

Table 3 Best Fuel costs- CELD solution for Case 1

\begin{tabular}{|c|c|c|}
\hline Variable & RCGA [42] & $\begin{array}{c}\text { Proposed } \\
\text { Algorithm DRN- } \\
\text { PSO }\end{array}$ \\
\hline $\mathrm{PG}_{1}$ (per unit) & 0.1727 & 0.1764 \\
\hline $\mathrm{PG}_{2}$ (per unit) & 0.3966 & 0.2852 \\
\hline $\mathrm{PG}_{3}$ (per unit) & 0.5679 & 0.4691 \\
\hline $\mathrm{PG}_{4}$ (per unit) & 1.1079 & 0.8981 \\
\hline $\mathrm{PG}_{5}$ (per unit) & 0.2194 & 0.6350 \\
\hline $\mathrm{PG}_{6}$ (per unit) & 0.3949 & 0.3029 \\
\hline Mean (Fuel cost) \$/hr & 623.3722 & 602.2351 \\
\hline Best (Fuel cost) \$/hr & 615.5482 & 591.1517 \\
\hline Worst (Fuel cost) \$/hr & 634.9026 & 619.1436 \\
\hline Standard-deviation & 5.7289 & 6.0778 \\
\hline $\begin{array}{c}\text { Emission at best fuel costs } \\
\text { ton/hr }\end{array}$ & 0.2285 & 0.215 \\
\hline
\end{tabular}

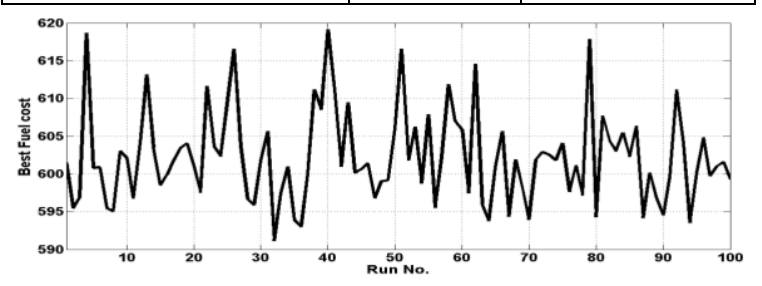

Fig. 3 Best fuel cost against runs number for Case 1

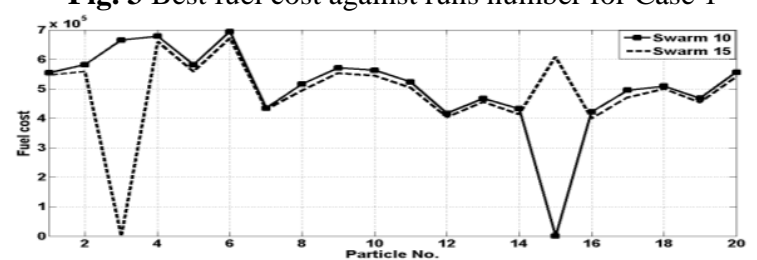

Fig. 4 Swarm 10 against swarm 15 for case 1

\subsubsection{Case 2: Emission minimization}

Case 2 considers the emission minimization only as a single objective. Table 4 shows that the fuel costs are increased to $681.87 \$ / \mathrm{hr}$ while the pollutant emission is reduced to the level of $0.1947 \mathrm{ton} / \mathrm{hr}$. In terms of the control variable settings, different security levels are obtained, especially from generators 1, 2 and 3. It is obvious that the obtained fuel costs for Case 2 using the proposed DRN-PSO algorithm are competitive compared to that obtained using RCGA. The convergence characteristics of case 2 are shown in Fig.s 5, 6. These Fig.s show the robust performance with fast convergence to the optimal solution at acceptable levels of standard deviations in the range of 0.0052 .

Table 4 Best emission CELD solution for Case 2

\begin{tabular}{|c|c|c|}
\hline Variables & RCGA [42] & Proposed approach \\
\hline $\boldsymbol{P}_{\boldsymbol{g}_{\mathbf{1}}}$ (per unit) & 0.3969 & 0.3950 \\
\hline $\boldsymbol{P}_{\boldsymbol{g}_{\mathbf{2}}}$ (per unit) & 0.4566 & 0.5331 \\
\hline $\boldsymbol{P}_{\boldsymbol{g}_{\mathbf{3}}}$ (per unit) & 0.6015 & 0.5718 \\
\hline $\boldsymbol{P}_{\boldsymbol{g}_{\mathbf{4}}}$ (per unit) & 0.3853 & 0.4075 \\
\hline $\boldsymbol{P}_{\boldsymbol{g}_{\mathbf{5}}}$ (per unit) & 0.5366 & 0.5671 \\
\hline $\boldsymbol{P}_{\boldsymbol{g}_{\mathbf{6}}}$ (per unit) & 0.5064 & 0.4682 \\
\hline Mean (Emission) ton/hr & 0.2018 & 0.2026 \\
\hline Best (Emission) & 0.1932 & 0.1947 \\
\hline Worst (Emission) & 0.2194 & 0.2247 \\
\hline Standard-deviation & 0.0056 & 0.0052 \\
\hline Fuel costs \$/hr & 691.3766 & 681.87 \\
\hline
\end{tabular}

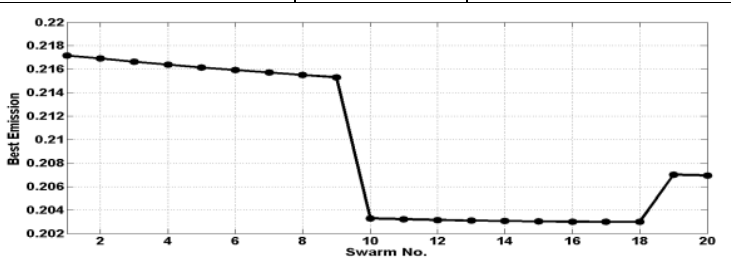

Fig. 5 Best particle in each swarm Case 2

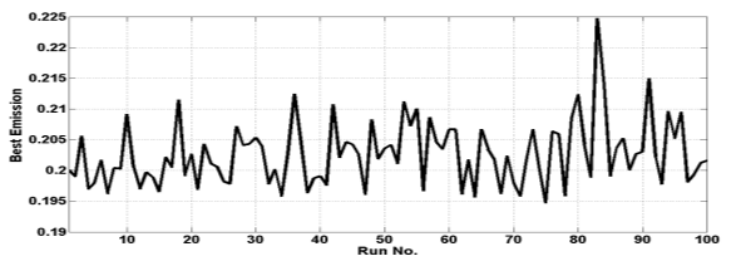

Fig. 6 Best particle against run number for Case 2

\subsubsection{Comparative studies for single objective categories}

Table 5 summarizes the comparison results between the proposed DRN-PSO against several optimization techniques for cases 1 and 2 of CELD problem. The use of the proposed method leads to responsible economical solutions of $591.1517 \$ / \mathrm{hr}$ and 681.87 $\$ / \mathrm{hr}$ for cases 1 and 2 , respectively. The corresponding emission levels are 0.215 and 0.1947 ton $/ \mathrm{hr}$. Previous results prove that the use of the proposed DRN-PSO algorithm leads to more economical compromised solutions compared to others at acceptable levels of emissions. 
Table 5 Comparison of different methods for compromised solutions for Cases 1 and 2

\begin{tabular}{|c|c|c|c|c|}
\hline \multirow{2}{*}{ Algorithm } & \multicolumn{2}{|c|}{ Case 1 } & \multicolumn{2}{c|}{ Case 2 } \\
\cline { 2 - 5 } & Cost $(\$ / \mathrm{h})$ & $\begin{array}{c}\text { Emissi } \\
\text { on } \\
(\text { Ton/h) }\end{array}$ & Cost $(\$ / \mathrm{h})$ & $\begin{array}{c}\text { Emission } \\
\text { (Ton/h) }\end{array}$ \\
\hline NSGA [40] & 600.3100 & 0.2238 & 633.8300 & 0.1946 \\
\hline NPGA [40] & 600.2200 & 0.2206 & 636.0400 & 0.1943 \\
\hline SPEA [40] & 600.3400 & 0.2241 & 640.4200 & 0.1942 \\
\hline FCPSO [18] & 600.1300 & 0.2223 & 638.3577 & 0.1942 \\
\hline MSFLA [41] & 600.1114 & 0.2221 & 638.2425 & 0.1942 \\
\hline RCGA [42] & 611.6935 & 0.2285 & 648.5301 & 0.1932 \\
\hline $\begin{array}{c}\text { Proposed } \\
\text { Approach }\end{array}$ & 591.1517 & 0.215 & 681.87 & 0.1947 \\
\hline
\end{tabular}

\subsubsection{Comparative studies for multiobjective problem}

\subsubsection{Case 3: Using equal weighting factors}

Returning back to Eq. (11) and using equal weighting factors is a multiobjective case that needs to be tested.

Table 6 shows the compromised solution using the proposed multiobjective version of DRN-PSO method for case 3 . The obtained results are compared to other optimization algorithms. The performance of multiobjective version of the proposed DRN-PSO model is shown in Fig.s 7-8. It is proven that, the proposed method has good convergence characteristics with robust solution.

Table 6 Comparison of different methods for the best compromise solution

\begin{tabular}{|c|c|c|}
\hline Algorithm & $\begin{array}{c}\text { Emission } \\
\text { (Ton/h) }\end{array}$ & Cost(\$/h) \\
\hline SPEA [40] & 0.2004 & 610.3 \\
\hline NSGA [40] & 0.2041 & 606.03 \\
\hline NPGA [40] & 0.2015 & 608.90 \\
\hline MSFLA [41] & 0.2006 & 610.0783 \\
\hline RCGA [42] & 0.2159 & 578.8774 \\
\hline Proposed approach for Case 3 & 0.2680 & 590.3951 \\
\hline
\end{tabular}

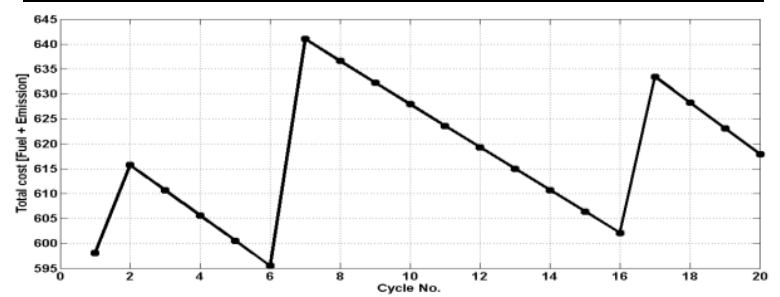

Fig. 7 Best particle against swarm number for case 3

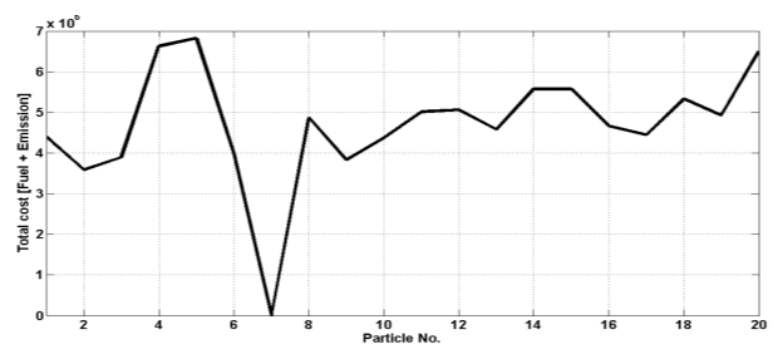

Fig. 8 Final swarm for Case 3

5.3.4.2 Case 4: Using weighted sum approach for different weighting factors

Table7 shows the compromised CELD solution solved by the proposed DRN-PSO compared to RCGA for Case 4 using different weighting factors (in the range from 10\%-90\%), respectively. This table shows that the best compromise solution is $588.8579 \$ / \mathrm{hr}$ at pollutant emission level of $0.2681 \mathrm{ton} / \mathrm{hr}$. It is proven that: the obtained results are competitive compared to those obtained by RCGA as shown in Table7. Thus, the proposed DRN-PSO method can be considered as an efficient promising method to solve non-linear optimization problems. The joint solutions of CELD problem for Case 4 prove the well distribution solutions which are successively obtained using the proposed DRNPSO method.

Table7 Joint CELD solution Case 4 for different weighting factors using DRN-PSO compared to RCGA

\begin{tabular}{|c|c|c|c|c|c|}
\hline \multicolumn{2}{|c|}{$\begin{array}{l}\text { Weightin } \\
\text { g factors }\end{array}$} & \multicolumn{2}{c|}{ RCGA [42] } & \multicolumn{2}{c|}{ Proposed DRN-PSO } \\
\hline $\boldsymbol{w}_{\mathbf{1}}$ & $\boldsymbol{w}_{\mathbf{2}}$ & Fuel Cost & Emission & $\begin{array}{c}\text { Fuel } \\
\text { Cost }\end{array}$ & Emission \\
\hline 0.9 & 0.1 & 589.9724 & 0.2245 & 589.5156 & 0.2681 \\
\hline 0.8 & 0.2 & 590.2386 & 0.2225 & 591.2503 & 0.2680 \\
\hline 0.7 & 0.3 & 589.6692 & 0.2160 & 591.2831 & 0.2680 \\
\hline 0.6 & 0.4 & 590.0404 & 0.2207 & 589.7860 & 0.2680 \\
\hline 0.5 & 0.5 & 590.0163 & 0.2155 & 588.8579 & 0.2681 \\
\hline 0.4 & 0.6 & 590.9255 & 0.2147 & 589.9727 & 0.2681 \\
\hline 0.3 & 0.7 & 592.1758 & 0.2197 & 592.0996 & 0.2681 \\
\hline 0.2 & 0.8 & 590.6076 & 0.2191 & 593.0636 & 0.2681 \\
\hline 0.1 & 0.9 & 591.0886 & 0.2226 & 593.0959 & 0.2680 \\
\hline
\end{tabular}

\section{CONCLUSIONS}

This paper is concerned with nonlinear constrained economic dispatch problem to enhance the operation of power plants and to help for building up effective generating management plans. This paper investigated an improved search algorithm based on the particle swarm optimization known multiobjective DRN-PSO for non-smooth constrained economic dispatch problems. Experiments were conducted on IEEE 30-bus and compared to other optimization techniques that reported in the literature. The obtained results demonstrate the superiority of the proposed DRN-PSO compared to other optimization techniques that reported in the literature. The proposed algorithm improves the economic issue as well as enhancing the power system operation in 
the technical point of view at acceptable levels of emissions. So, it can be considered as a promising alternative algorithm for solving problems in practical large scale power systems

\section{REFERENCES}

[1] A. J. Wood and B. F. Wollenbergy, Power Generation, Operation, and Control. New York: Wiley, 1984.

[2] J. Zhu, "Optimization of Power System Operation," A John Wiley \& Sons, Inc. Publication, IEEE 2009.

[3] I. G. Damousis, A. G. Bakirtzis, , and P. S. Dokopoulos," Network-Constrained Economic Dispatch Using Real-Coded Genetic Algorithm ," IEEE transactions on power systems, vol. 18, no. 1, February 2003, pp.198-205

[4] M.A. Abido, "A niched Pareto genetic algorithm for multiobjective environmental/economic dispatch," Electrical Power and Energy Systems, 25 (2009): 97-105.

[5] B. Gjorgiev, M. Cepin, "A multi-objective optimization based solution for the combined economic-environmental power dispatch problem," Engineering Applications of Artificial Intelligence, 26(2013): 417-429.

[6] L.H. Wu, Y.N. Wang, X.F. Yuan,, S.W. Zhou, "Environmental/economic power dispatch problem using multi-objective differential evolution algorithm," Electric Power Systems Research, 80 (2010):1171-1181.

[7] A.A. Abou El-Ela, M.A. Bishr, S.M. Allam, R.A. El-Sehiemy, "An emergency power system control based on the multi-stage fuzzy based procedure," Electric Power Systems Research Volume 77, Issues 5-6, April 2007, pp. 421429

[8] K. T. Chaturvedi, M. Pandit and L. Srivastava, "Self-Organization Hierarchical Particle Swarm Optimization for Nonconvex Economic Dispatch", IEEE Trans. on Power Systems, Vol. 23, No. 3, pp. 1079-1087, August 2008.

[9] A.P. Engelbrecht, Fundamentals of Computational Swarm Intelligence, John Wiley \& Sons, 2006.

[10] Kennedy, R.C. Eberhart, Y. Shi, Swarm Intelligence, Morgan Kaufmann, San Francisco, CA, 2001.

[11] M.R. AlRashidi, M.E. El-Hawary, A survey of particle swarm optimization applications in electric power systems, IEEE Transactions on Evolutionary Computation 14 (4) (2009) 913918.

[12] A. Banks, J. Vincent, C. Anyakoha, A review of particle swarm optimization. Part II: hybridization, combinatorial, multicriteria and constrained optimization and indicative applications, Natural Computing: An International Journal 7 (2) (2008) 109-124.

[13] Y. Del Valle, G.K. Venayagamoorthy, S. Mohagheghi, J.C. Hernandez, R.G. Harley, Particle swarm optimization: Basic concepts, variants and applications in power systems, IEEE Transactions on Evolutionary Computation 12 (2) (2008) 171-195.

[14] R.C. Eberhart, Y. Shi, Guest editorial, IEEE Transactions on evolutionary computationsspecial issue particle swarm optimization, IEEE Transactions on Evolutionary Computation 8 (3) (2004) 201-203.

[15] J. Kennedy, R. Mendes, Population structure and particle swarm performance, in: Proceedings of IEEE Congress on Evolutionary Computation, Honolulu, HI, 2002, pp. 16711676.

[16] X.D. Li, A.P. Engelbrecht, Particle swarm optimization: an introduction and its recent developments, in: Proceedings of Genetic Evolutionary Computation Conference, 2007, pp. 3391-3414.

[17] Ali R. Yildiz, A novel particle swarm optimization approach for product design and manufacturing, International Journal of Advanced Manufacturing Technology 40 (5-6) (2009) 617-628.

[18] S. Agrawal, B. K. Panigrahi and M. K. Tiwari, "Multiobjective Particle Swarm Algorithm With Fuzzy Clustering for Electrical Power Dispatch", IEEE Trans. on Evolutionary Computation. Vol. 12, No. 5, pp. 529-541, Oct.2008.

[19] K. Meng, H. G. Wang; Z. Y. Dong; K. P. Wong," Quantum-Inspired Particle Swarm Optimization for Valve-Point Economic Load Dispatch", Power Systems IEEE Transactions, Vol. 25, No. 1, pp. 215-222, 2010.

[20] A.Bhattacharya, P.K. Chattopadhyay, "Hybrid Differential Evolution With BiogeographyBased Optimization for Solution of Economic Load Dispatch", IEEE Trans. on Power Systems, Vol. 25, No. 4, pp. 1955-1964, 2010.

[21] A. Rabiee, B.Mohammadi-Ivatloo, M.Ehsan, " Discussion of Hybrid Differential Evolution With Biogeography-Based Optimization for Solution of Economic Load Dispatch", IEEE Trans. on Power Systems, Vol. 27, No. 1, pp.574-, 2012.

[22] A. Bhattacharya, and P. K. Chattopadhyay," Biogeography-based optimization for different economic load dispatch problems," IEEE Transactions on power systems, Vol. 25, No. 2, May 2010, pp.1064-1077.

[23] W. M. Lin, F. S. Cheng, and M. T. Tsay,“An improved Tabu search for economic dispatch 
with multiple minima,'IEEE Trans. Power Syst., vol. 17, pp. 108-112, Feb. 2002.

[24] S. Pothiya, I. Ngamroo, W. Kongprawechnon, "Ant colony optimization for economic dispatch problem with non-smooth cost functions," International Journal of Electrical power and Energy SystemVol. 32, pp. 478-487, June 2010.

[25] B. Shaw, V. Mukherjee, S.P. Ghoshal "Solution of economic dispatch problems by seeker optimization algorithm, "Expert Systems with Applications, Volume 39, Issue 1, pp. 508-519 January 2012.

[26] A.E. Eiben, R. Hinterding, Z. Michalewicz, "Parameter Control in Evolutionary Algorithms", IEEE Transactions on Evolutionary Computation, Volume 3, Issue 2, Page(s): 124 - 141, July 1999

[27] E. K. P. Chong, S. H. Zak, "An Introduction to Optimization", Second Edition, Wiley Interscience Series in Discrete Mathematic And Optimization, 2001.

[28] R. A. El - Sehiemy, M. B. Shafiq, A. R. Azmy, "An Enhanced Seeker Optimization Algorithm For constrained Optimal Power Dispatch Problem," Fifteenth International Middle East Power Systems Conference (MEPCON'12), Alexenderai University, Egypt, Dec. 2012.

[29] X. Yang, S. S. S. Hosseini, A. H. Gandomi, "Firefly Algorithm for solving non-convex economic dispatch problems with valve loading effect ,"Applied Soft Computing 12 (2012) 180-1186.

[30] A. A. El-Ela, M Bishr, S Allam, R El-Sehiemy, "Optimal preventive control actions using multi-objective fuzzy linear programming technique," Electric power systems research 74 (1), 147-155.

[31] E. S. Peer, F. Van den Bergh, A. P. Engelbrecht, Using Neighborhoods with the guaranteed Convergence PSO, 200, IEEE, pp. 235-242

[32] P.N. Suganthan, Particle swarm optimizer with neighborhood operator, in: Proceedings of IEEE Congress on Evolutionary Computation, 1999, pp.1958-1962.
[33] X. Hu and R. C. Eberhart, "Multiobjective optimization using dynamic neighborhood particle swarm optimization," in Proc. Congr. Evol. Comput., 2002, pp. 1677-1681.

[34] Wei Jian, Yuncan Xue, and Jixin Qian,"Improved Particle swarm optimization algorithms study based on the neighborhoods topologies", the 30th Annual Conference of the IEEE Industrial Electronics Society, November 2-6, 2004, Busan, Korea.

[35] J. Kennedy, Small worlds and mega-minds: effects of neighborhood topology on particle swarm performance, in: Proceedings of IEEE Congress on Evolutionary Computation, 1999, pp. 1391-1938.

[36] R. Mendes, J. Kennedy, J. Neves, The fully informed particle swarm: simpler, maybe better, IEEE Transactions on Evolutionary Computation 8 (3) (2004) 204-210.

[37] T. Peram, K. Veeramachaneni, C.K. Mchan, Fitness-distance-ratio based particle swarm optimization, in: Proceedings of the IEEE Swarm Intelligence Symposium, 2003, pp. 174181.

[38] S. Ghosh, S. Das, D. Kundu, K. Suresh, A. Abraham, Inter-particle communication and search-dynamics of lbest particle swarm optimizers: an analysis,Information Sciences 182 (1) (2012) 156-168.

[39] Washington University Website: www.ee.washington.edu/research/pstca/

[40] M.A.Abido, Multiobjective evolutionary algorithms for electric power dispatch problem. IEEE Trans. on Evolutionary Computation, 10 (2006): 315-329.

[41] A. S. Reddy and K. Vaisakh, "Economic Emission Load Dispatch by Modified Shuffled Frog Leaping Algorithm," International Journal of Computer Applications, 31 (2011): 58-65.

[42] Ragab A. El-Sehiemy, Mostafa Abdelkhalik ElHosseini and Aboul Ella Hassanien, "Multiobjective Real-Coded Genetic Algorithm for Economic/Environmental Dispatch Problem," Studies in Informatics and Control, Vol. 22, No. 2, June 2013, pp. 113-122. 